\title{
Onset of turbulence in a regular porous medium: An experimental study
}

\author{
N. A. Horton and D. Pokrajac \\ Department of Engineering, University of Aberdeen, Fraser Noble Building, \\ Aberdeen AB24 3UE, United Kingdom
}

(Received 23 September 2008; accepted 8 December 2008; published online 16 April 2009)

\begin{abstract}
This paper presents the results from an experimental study on turbulent flows through a regular porous matrix of spheres packed in a cubic arrangement. The special pore geometry made it possible to measure the velocity within individual pores, using the ultrasonic velocity profiler (UVP). The UVP recorded the time series of the average cross-sectional velocity at a series of points along the pore. The measurements were performed for various hydraulic gradients resulting in a pore Reynolds number between 70 and 430. Three different regimes were detected: unsteady laminar, transition to turbulence, and turbulent. An additional measurement was performed using particle image velocimetry (PIV), which recorded two velocity components within a window which covered only the widest pore section. The PIV test had a Reynolds number of 370, which corresponds to transitional flow just before the onset of turbulence. The PIV result revealed the spatial distribution of mean velocities and turbulence intensities, as well as the shapes of coherent flow structures during significant turbulent events. (C) 2009 American Institute of Physics.
\end{abstract}

[DOI: 10.1063/1.3091944]

\section{BACKGROUND}

The study of fluid flows through porous media is important for a wide range of scientific and engineering applications. Oil recovery, heat exchangers, and filtration systems are all practical examples that are important from an industrial point of view. The understanding of flow through porous media is also necessary for civil and geotechnical engineering where fluid flows through earth and rock structures can impact construction projects or cause flooding.

Research into porous media flow has a long history. The original work in this area was done by Henry Darcy in the 19th century when he established the well known relationship between the unit flux, $u_{D}$ and the hydraulic gradient, $\Delta H / L$,

$$
u_{D}=-K \frac{\Delta H}{L},
$$

where $K$ is the hydraulic conductivity of the porous medium for a particular fluid. The hydraulic conductivity can be expressed as $K=k g / \nu$, where $k$ is the intrinsic permeability of the porous medium and $\nu$ is the kinematic viscosity of the fluid.

Darcy's law is a "volume-averaged" expression relating the bulk hydraulic gradient to the bulk flux and its applicability depends on the pore-scale hydrodynamics being averaged. This also applies for Forchheimer's equation,

$$
-\frac{\Delta H}{L}=\alpha u_{D}+\beta \rho u_{D}^{2},
$$

where $\alpha$ is the reciprocal hydraulic conductivity and $\beta$ is known as the "inertial" parameter. Both $\alpha$ and $\beta$ depend on the porosity of the material.

Researchers such as Kozeny, Carman, Ergun, ${ }^{1}$ and Ward ${ }^{2}$ also published well known studies that attempt to extend the
Darcy and Forchheimer laws. Dybbs and Edwards ${ }^{3}$ performed a study that investigated flow through a bed of rods in a regular, hexagonal packing. Using laser anenometry and visualization studies over a wide range of Reynolds numbers, they highlighted the existence of Darcian, steady-laminar (inertial), unsteady-laminar, and turbulent regimes. Many of the studies since this time referenced Dybbs and Edwards and also cited the existence of four regimes. However, in these studies, a pre-Darcy regime is often highlighted (i.e., Fand et $a l^{4}$ ) and the inertial regime is presented as a transition region between linear and nonlinear flow.

To explain this further, let us consider the Navier-Stokes equation averaged over the volume, $A d x$, of a single representative pore of the porous medium ( $A$ is the cross-sectional area of the pore). With progressive increases in the pore velocity the following regimes can be distinguished.

(1) Regime 1. For small Reynolds numbers inertia is negligible and contributions to the Navier-Stokes equations come from the hydraulic gradient and the skin friction (viscous shear stress at the pore wall). The skin friction is proportional to the velocity gradient across the cross section of the pore, so the local fluid velocity scales with the (spatially averaged) hydraulic gradient. Averaging this relationship over a representative set of pores [i.e., representative elementary volume (REV)] yields Darcy's law.

(2) Regime 2. The flow in this regime is steady and laminar. Contributions from the inertial term (advective acceleration) increase with the Reynolds number and once the Reynolds number is sufficiently high, the nonlinear inertial term starts to affect momentum balance. This results in a nonlinear macroscopic resistance law such as Forchheimer's equation.

(3) Regime 3. The advective acceleration term becomes suf- 
Micro-Scale

\begin{tabular}{|c|c|c|c|c|}
\hline \multicolumn{3}{|c|}{ LAMINAR } & TRANSITION & TURBULENT \\
\hline \multicolumn{2}{|c|}{ STEADY } & UNSTEADY & & \\
\hline Linear & $\begin{array}{l}\text { Non } \\
\text { Linear }\end{array}$ & & & \\
\hline
\end{tabular}

\section{Macro-Scale (Bulk)}

\begin{tabular}{|l|l|l|}
\hline DARCY FORCHHEIMER & TURBULENT \\
\hline
\end{tabular}

FIG. 1. Comparison of micro- and macroscale regime classifications.

ficiently large to cause flow instabilities, which grow as the Reynolds number increases. In the experiments by Dybbs and Edwards, these instabilities were observed as pulsations of the dye streamers. These pulsations were periodic and grew until eventually dispersing the dye as it left the observation window.

(4) Regime 4. High Reynolds numbers lead to further flow instabilities and turbulence production. This regime was classified as unsteady and chaotic by Dybbs and Edwards. It was noted that at this stage, the flow was not laminar and qualitatively resembled the turbulent flow identified by Reynolds in his classic work on pipe flow transition.

For regimes 3 and 4 the macroscopic relationship between the pore velocity and the volume-averaged hydraulic gradient is obtained by averaging over the REV and over time. It has the same form as Forchheimer's equation, but the coefficients may be different due to the different natures of the local flow within the pore.

Dybbs and Edwards ${ }^{3}$ stated that regime 2 develops in parallel with the boundary layers that form near the solid boundaries. It was highlighted that the growth of these boundary layers eventually leads to the presence of an "inertial core." In random porous media, detecting this inertial core is difficult because it requires a detailed measurement of local velocity within irregular pores.

There are two important issues related to the flow regimes presented in literature. The first issue is the scale of observation used for classifying flow regimes. In directly measuring flow variables, both Dybbs and Edwards ${ }^{3}$ and Seguin et al. $^{5}$ presented a description of the flow regimes which were based on microscale properties. Alternatively, studies that are based on assessments of bulk resistance, such as that of Fand et ll. $^{4}$ and Kececioglu and Jiang ${ }^{6}$ used a more traditional porous media approach in classifying the flow regimes from a macroscale perspective. It is important to recognize this difference as confusion can arise when attempting to compare these results. Figure 1 shows the relationship between the flow regimes inferred from the following two types of experiments:

(a) pore-scale studies that attempt to resolve the various "point" terms in the Navier-Stokes equations;

(b) bulk-scale studies that consider the relationship be-

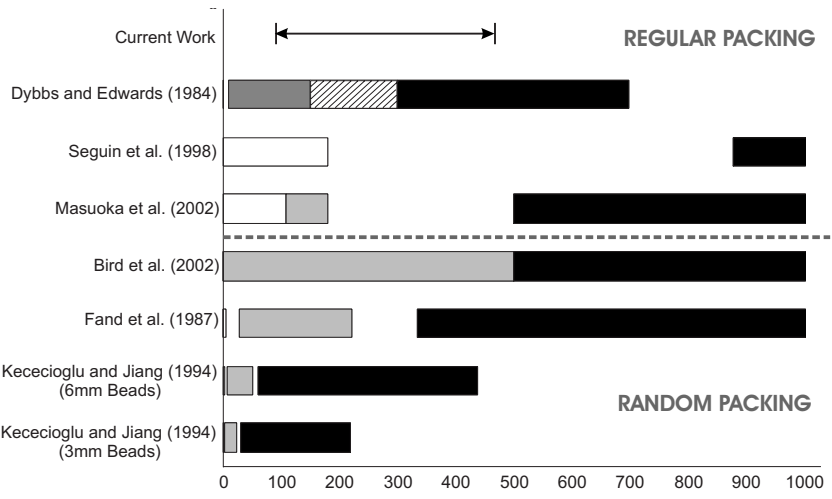

FIG. 2. Limits of the porous media flow regimes. Microscale: (i) steady linear laminar (white), (ii) steady, nonlinear laminar-inertial core (dark gray), (iii) unsteady laminar (hatched), and (iv) turbulent (black). Macroscale: (i) Darcian (white), (ii) Forchheimer (light gray), and (iii) turbulent (black). In Ref. 5 the white section is described as the stable laminar regime. The dashed line separates regular (above the line) and random (below the line) packing of the porous media.

tween volume-averaged components of the NavierStokes equations and the bulk hydraulic gradient. These studies mainly consider how the bulk hydraulic gradient relates to the bulk velocity across different flow regimes, defined in a volume-averaged sense.

The second issue relates to the identification of regime limits. Since a porous medium is a bundle of tubesalthough very complex ones-we expect some kind of Reynolds number to serve as a criterion for detecting the regime limits. Various definitions of the relevant Reynolds number exists in literature and conversion between them can be difficult or impossible if some of the experimental parameters are missing. In this study we use the conventionally defined Reynolds number,

$$
\operatorname{Re}=\frac{\rho U d}{\mu},
$$

where $\operatorname{Re}$ is the pore Reynolds number, $U$ is the pore velocity (later defined as the double-averaged fluid velocity), $d$ is a characteristic length of the porous media (the bead or tube diameter), $\rho$ is the density of the fluid, and $\mu$ is the viscosity of the fluid.

Figure 2 is an overview of the regime limits found in literature, based on the Reynolds number of expression (3). The values of $U$ and $d$ were recalculated where necessary.

In comparing the Reynolds numbers when various flow regimes are expected to occur, it is clear that there is very little overall agreement. Seguin et al. $^{5}$ presented an analogous comparison, which worked well where the porous media were similar. However it was highlighted that strictly speaking, due to the very particular porous geometries used, "the values of the Reynolds numbers corresponding to the different transitions cannot be extrapolated to any other porous media." This is very obvious from Fig. 1 as for randomly packed materials, the onset of turbulent flow occurs much earlier than in regular beds. The Reynolds number, marking the transition to turbulent regimes, can significantly vary even when comparing similar porous materials. 


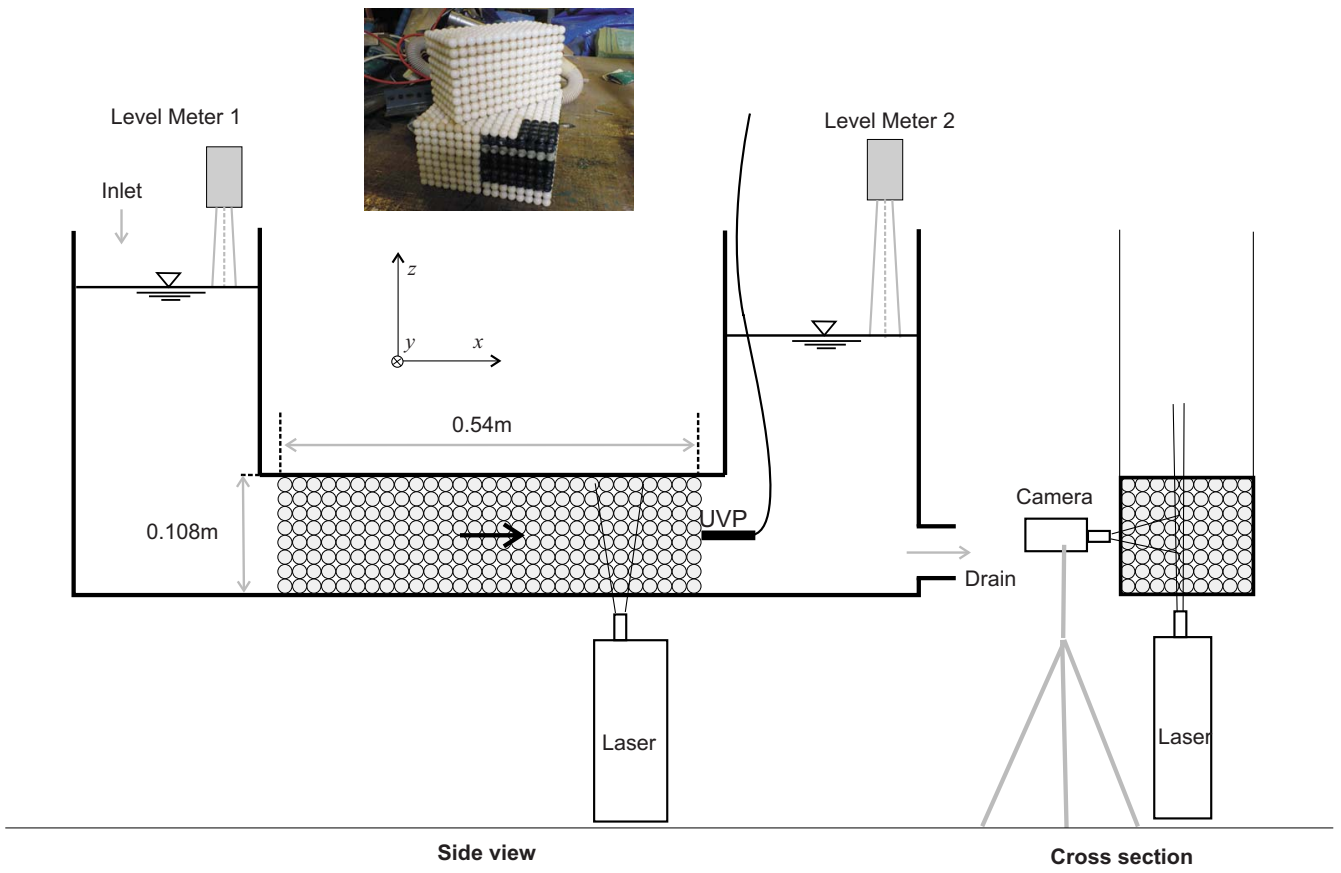

FIG. 3. (Color online) Experimental apparatus. The porous medium consists of $12 \mathrm{~mm}$ diameter spheres packed in a cubic arrangement (inset).

It is the individuality of each porous medium that has led some researchers (e.g., Dybbs and Edwards ${ }^{3}$ ) to focus on regular, artificial media to establish universal regime limits. However, even in such porous media, various flow regimes are detected mainly by flow visualization so that the associated velocity statistics are not known. This paper presents an experimental investigation into velocity statistics that characterize flow regimes in a regular porous medium prior to and after the onset of turbulence. In using velocity data measured directly in the pore we seek to clarify the uncertainty surrounding porous media flow regimes, and particularly turbulence generation and structure.

\section{EXPERIMENTAL SETUP}

A series of experiments were carried out with steady (on average) flow through a regular porous medium made of uniform size spheres. The main objective was to conduct representative measurements of pore velocities across a wide range of flow rates that span the flow regimes reported earlier. A particular packing arrangement allowed us to measure the variation of pore velocity along several sphere diameters. This section describes the experimental rig, provides the details of the experimental techniques and lists the experimental conditions.

\section{A. Apparatus and porous medium}

The experimental rig consists of two main tanks connected by a closed rectangular channel that houses the porous medium (Fig. 3). A further small tank with a side weir was attached to each main tank to maintain constant water levels during the experiments.

The porous medium was made of $12 \mathrm{~mm}$ diameter polyacetal (delrin) plastic spheres with $\pm 25 \mu \mathrm{m}$ tolerance and $\pm 25 \mu \mathrm{m}$ sphericity. These were packed in a regular cubic pattern to create a pore space of straight tubes. The cross section of the pores varied between a $12 \times 12 \mathrm{~mm}^{2}$ square and a diamond-shaped throat with a minimum pore diameter of $5 \mathrm{~mm}$. A porous "brick" measuring $540 \times 96 \times 108 \mathrm{~mm}^{3}$ was formed by gluing the spheres together. The medium was then placed in the rectangular channel, which was designed to fit tightly along its width and height.

\section{B. Velocity measurement}

The main set of measurements was made using the ultrasonic velocity profiler (UVP), which is capable of recording the cross-sectional velocity along a pore and hence providing relevant velocity statistics at the scale of a single pore. A further experiment, involving particle image velocimetry (PIV), was performed in order to provide information about the spatial flow structure.

\section{Ultrasonic velocity profiler}

The Met-Flow SA 2000 UVP is a nonintrusive velocity measurement system suitable for data acquisition within the porous medium constructed here (Manes et $a l .{ }^{7}$ ). The pulse of ultrasound which is emitted by the UVP probe travels along the measurement axis. When the ultrasound pulse hits any seeding particle which is moving with the flow, a portion of the ultrasound's energy is scattered and echoes back. At this point the probe has switched to a receiving or "listening" phase. By measuring the Doppler shift of the frequency and the time it takes for the echo to reach the transducer the UVP can calculate the position and velocity of the particle.

The UVP probe was positioned in the downstream tank facing the pore of interest (Fig. 3). A neutrally buoyant seeding was fed directly into the upstream brick of beads via a thin tube ( $5 \mathrm{~mm}$ outer diameter). Sensitivity tests were performed to establish the dependence of the measured veloci- 


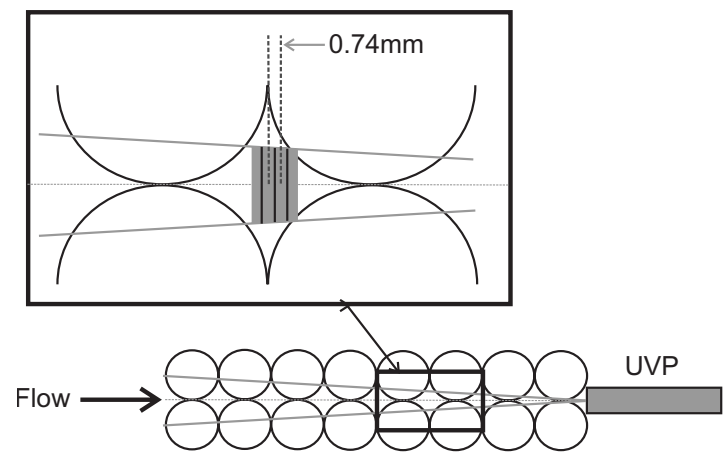

FIG. 4. Sketch of the $4 \mathrm{MHz}$ UVP probe positioned in the downstream tank, facing the pore of interest

ties on the position of the probe. No measurable wall effect was detected, i.e., the measured velocities were found to be negligibly different at all horizontal and vertical pores along the central axis. Thus the data were acquired at the center of the porous block. For the majority of these tests the transducer was positioned such that the distance from the probe face to the nearest layer of beads was $x_{\mathrm{pf}}=10 \mathrm{~mm}$. However this was not always practical and therefore some tests were performed with $x_{\mathrm{pf}}=0 \mathrm{~mm}$ (as shown in Fig. 4). The measurements were also found to be insensitive to this distance.

Figure 4 illustrates the $4 \mathrm{MHz}$ UVP probe that was used for the experiments. The probe has a physical diameter of 8 $\mathrm{mm}$, a working diameter of $5 \mathrm{~mm}$ and a beam divergence of $2.2^{\circ}$. It recorded data at 128 locations (channels) over a 95 $\mathrm{mm}$ measurement window, with an average sampling frequency of $30 \mathrm{~Hz}$. The disk-shaped sampling volumes are at $0.74 \mathrm{~mm}$ intervals. They are also $0.74 \mathrm{~mm}$ in length and are approximately $5 \mathrm{~mm}$ in diameter, although their exact size is dictated by the beam divergence. The relatively large diameter of the sampling volume is beneficial for this particular application as it means that the UVP measures the average velocity across the whole $5 \mathrm{~mm}$ diameter circle, inside the pore, i.e., providing a good approximation of the pore velocity. This was confirmed in a previous study (Manes et al. ${ }^{7}$ ) by comparison with velocities measured using PIV. The present study provides further confirmation by comparing UVP-measured velocities in the pore throats with those found from the total discharge through the apparatus (Fig. 6).

The ability of the UVP system to acquire time series results at many channels along the beam axis means that temporally and spatially averaged data can be calculated. The results were reliable between 5 and $90 \mathrm{~mm}$ from the probe although spatial averaging was performed over a somewhat smaller window, spanning over three whole spheres (between 48 and $84 \mathrm{~mm}$ from the probe).

\section{PIV}

The planar velocity field within the same pore space was measured using PIV. PIV is a nonintrusive measurement system which relies on capturing images of laser-illuminated seeding that is traveling with the flow. The system that was used in this study consists of a New Wave Research Solo III, double-pulsed Nd:YAG (yttrium aluminum garnet) laser and a FlowSense 2M digital camera, with a $60 \mathrm{~mm}$ Nikon AF Micro Nikkor fixed focal length lens. Over a very short time period (approximately equal to $1 \mathrm{~ms}$ ) the laser illuminates the measurement window twice, allowing the camera to record a pair of images. This is repeated at a sampling rate of 13.5 Hz. Neutrally buoyant, silver coated hollow glass spheres were used to seed the flow.

For the purpose of the PIV analysis the images are divided into rectangular sections called interrogation areas. The average particle displacement in each interrogation area is determined by the maximum cross-correlation coefficient of the light intensity in the two images. The analysis is repeated several times, each time with a smaller interrogation area: The displacement found in the previous pass is used to shift the subsequent interrogation areas to the most likely position of the particles in both images. This procedure is called adaptive correlation. In our analysis the size of the initial interrogation area was $128 \times 128$ pixels and the adaptive correlation was performed in two steps $(64 \times 64$ and $32 \times 32$ pixels). Dantec's DYNAMIC STUDIO software package was used for both laser/camera control and correlation analysis.

With reference to Fig. 3 the laser was placed beneath the test-section so that the light sheet shone up from below the flow. The light sheet was aligned with the streamwise axis near the center of the channel and the camera viewed the flow through the perpendicular pores. The instantaneous velocity field in the $x-z$ plane was measured through time, over the length of two pore spaces. The physical size of a pixel was $0.023 \mathrm{~mm}$, resulting in a final interrogation area size of $0.74 \mathrm{~mm}$. The time between two successive images was 2 $\mathrm{ms}$. To improve the resolution of the data points and optimize the use of the recorded seeding images, the interrogation areas were made to overlap by $50 \%$.

It is worth noting that the polyacetal beads used throughout the tests were manufactured such that they had a dull, matt finish. It was found that this was better suited for use with both the UVP and PIV systems; polished surfaces tended to cause reflections of ultrasound and laser light, giving much poorer data.

\section{Level measurement}

Obtaining accurate results was highly dependant on the accuracy of the head difference between the upstream and downstream tanks. Measurements of this difference were performed in two separate ways. First Vernier gauges with an accuracy of $0.02 \mathrm{~mm}$ were used. By taking a series of measurements the mean error was evaluated at around $0.05 \mathrm{~mm}$. The second method made use of HiTECH noncontact ultrasonic compact level transceivers. The transceivers are cylindrical in shape, having an outer housing that is $50 \mathrm{~mm}$ in diameter and $130 \mathrm{~mm}$ in length. In a similar manner to the UVP, the level transceiver emits and then receives an acoustic beam from any obstacle at which it is being directed-in this case the water surface within the tanks. Unlike the UVP these systems do not work with a dedicated computer and therefore required extensive calibration before the output voltages could be confidently converted to distance data. 
TABLE I. Experimental conditions: $u$ is the pore velocity measured by the UVP at a series of locations along a pore, $\bar{\theta}$ denotes time averaging ( $\theta$ is an arbitrary variable), and \langle\rangle denotes spatial averaging over the length of three spheres. The pore Reynolds number was calculated as $\operatorname{Re}=\langle\bar{u}\rangle d / \nu$ with $d$ $=5 \mathrm{~mm}$.

\begin{tabular}{ccccc}
\hline \hline & & $\langle\bar{u}\rangle$ & & \\
Test No. & Hydraulic gradient & $(\mathrm{mm} / \mathrm{s})$ & $\operatorname{Re}_{p}$ & Marker symbol \\
\hline 1 & 0.0012 & 20.5 & 67.4 & $\bullet$ \\
2 & 0.0027 & 33.5 & 110.3 & $\bigcirc$ \\
3 & 0.0036 & 52.5 & 172.8 & $\square$ \\
4 & 0.0079 & 74.4 & 244.6 & $\star$ \\
5 & 0.0082 & 73.9 & 243.2 & $\nabla$ \\
6 & 0.0095 & 84.4 & 277.7 & $\times$ \\
7 & 0.0110 & 95.4 & 313.9 & + \\
8 & 0.0130 & 105.1 & 344.9 & $\searrow$ \\
9 & 0.0184 & 132.4 & 438.5 & $\bullet$ \\
10 & 0.0176 & 133.3 & 435.5 & $\triangleright$ \\
\hline \hline
\end{tabular}

Good results were achieved for distances between 160 and $900 \mathrm{~mm}$ from the probe face. From the manufacturer's specifications, the transceivers achieve a repeatability of approximately $0.125 \mathrm{~mm}$. During the calibration it was found that the transceivers were limited to a measurement resolution of $0.125 \mathrm{~mm}$ and this resulted in an overall repeatability of 0.25 $\mathrm{mm}$. Nevertheless, good agreement was found between the slopes calculated with the Vernier gauges and those with the level transceivers.

\section{Experimental conditions}

A single experiment involved setting up a steady state flow, followed by the measurement of the hydraulic head difference between the main tanks and the velocity in the selected pore. An additional set of experiments were performed to measure the total discharge rather than the velocity.

For the UVP measurements the head difference was varied between 0.001 and $0.01 \mathrm{~m}$, resulting in hydraulic gradients between $1 / 833$ and $1 / 543$.

Table I lists the following data for each UVP experiment: the hydraulic gradients, the UVP-measured pore velocities averaged over time and the length of three spheres, the Reynolds numbers based on this pore velocity and the bead diameter, and the markers used to present experimental results in Sec. III of this paper.

The PIV measurement window covered only part of the active pore volume so the measured velocities could not be used for determining the pore velocity and Reynolds number. Instead they were evaluated from the discharge (measured volumetrically) and the streamwise variation of the pore velocity recorded by the UVP (please see Sec. III A). The pore velocity in the PIV measurement was $86 \mathrm{~mm} / \mathrm{s}$ and the pore Reynolds number was 370 . Therefore the experimental conditions approximately matched those of UVP Experiment 8. (a)

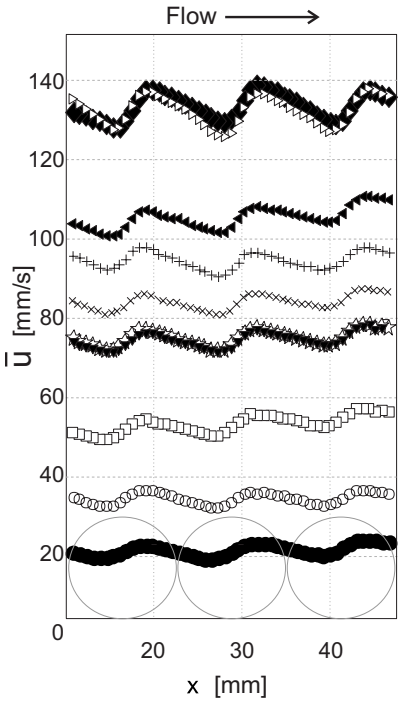

(b)

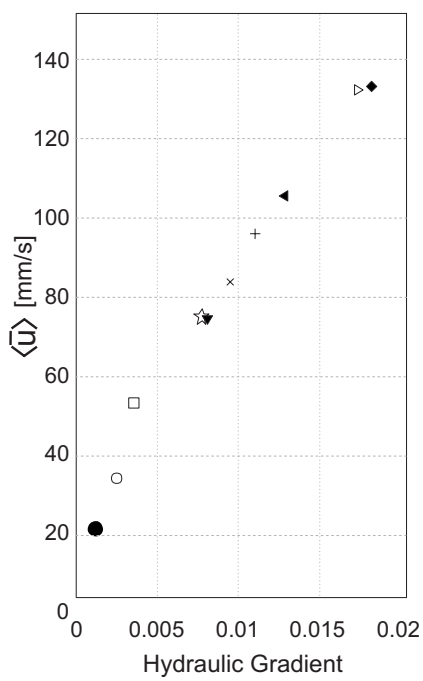

FIG. 5. (a) Time-averaged pore velocity $\bar{u}$ along the pore. (b) Doubleaveraged pore velocity $\langle\bar{u}\rangle$ for various hydraulic gradients (for the legend please refer to Table I).

\section{RESULTS AND DISCUSSION}

A coordinate axis in the flow direction, following the center of a pore, is denoted with $x$ and the instantaneous velocity in this $x$ direction, with $u$. To obtain macroscopic flow quantities, spatial averaging was applied to timeaveraged quantities resulting in double-averaged quantities. The spatial-averaging operator is denoted by \langle\rangle , e.g., the time-averaged velocity at a point is $\bar{u}$, and the doubleaveraged velocity is $\langle\bar{u}\rangle$. All spatially averaged quantities are obtained using a window which spanned over three spheres.

\section{A. Mean velocity and discharge}

Time-averaged streamwise velocity profiles measured using the UVP system, for tests with slopes between 0.0012 and 0.0184 are shown in Fig. 5. They confirm that reasonably uniform (on average) flow has been maintained well throughout the pore.

The wavy appearance of each profile is due to the flow accelerating and decelerating through the narrowest (throat) and widest regions of the pore space, with local maxima and minima occurring just downstream of each. In all tests the "waves" are asymmetric suggesting shorter flow converging and longer flow-diverging sections. The amplitude of the waves increases with the hydraulic gradient.

The relationship between the average pore velocity and hydraulic gradient can be used to evaluate the macroscopic resistance law. A good agreement with the experimental data shown in Fig. 5 was found for the following coefficients:

$$
-\frac{\Delta H}{L}=0.06\langle\bar{u}\rangle+0.57\langle\bar{u}\rangle^{2} \text {. }
$$

The UVP sampling volume does not cover the whole cross section of the pore but only its central part-a $5 \mathrm{~mm}$ diameter circle. To establish how the UVP-measured pore velocity relates to the bulk discharge, the velocity at the narrowest 


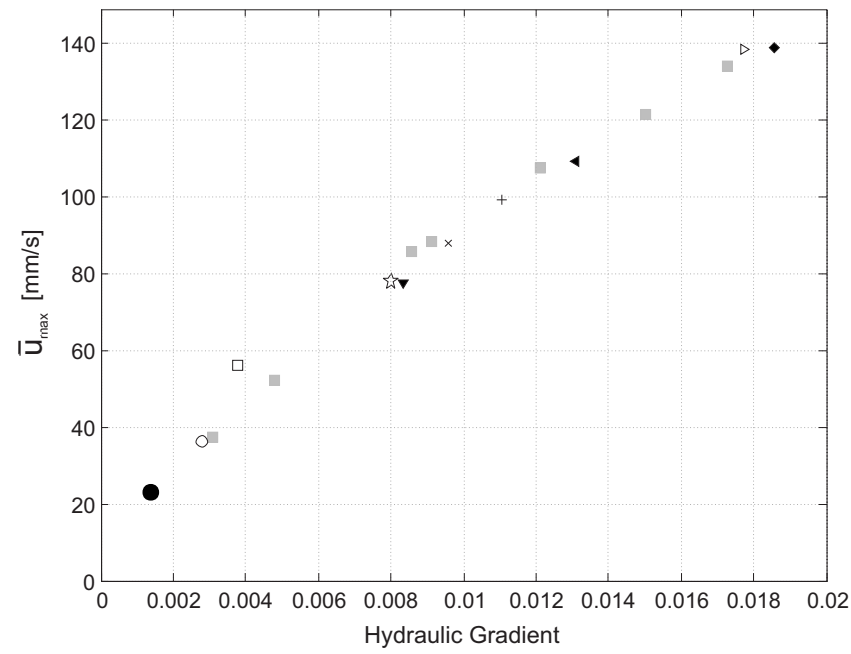

FIG. 6. Comparison between maximum velocities along the pore, $\bar{u}_{\max }$ and those found from discharge tests (gray squares).

cross section was calculated using direct measurements of bulk discharge and the area of the throat. Figure 6 shows these data (gray symbols) together with the spatial average, over three spheres, of the maximum UVP-measured velocity (black symbols). The results from the UVP tests agree very well with the discharge data confirming that the sampling volume of the UVP probe covers the whole active cross section of the pore, at least at the throat.

This finding, combined with the asymmetric and periodic shape of the velocity waves allows us to construct a conceptual model of the active flow tube or active core within the pore (Fig. 7). The core consists of a longer diverging section and a shorter converging section. The diverging section starts at the position of maximum velocity, slightly downstream of the pore throat, where the flow separates from the boundary. The diverging section ends in the region of reattachment, somewhat upstream of the next throat. After reattachment the fluid is forced through a short converging section by the geometry of the pore. The figure also shows the regions of low-momentum fluid trapped in the gaps between the spheres, above and below the flow tube. Where the active core flow has low velocity this fluid is expected to form a stable rotorlike vortex, whereas at higher velocities it

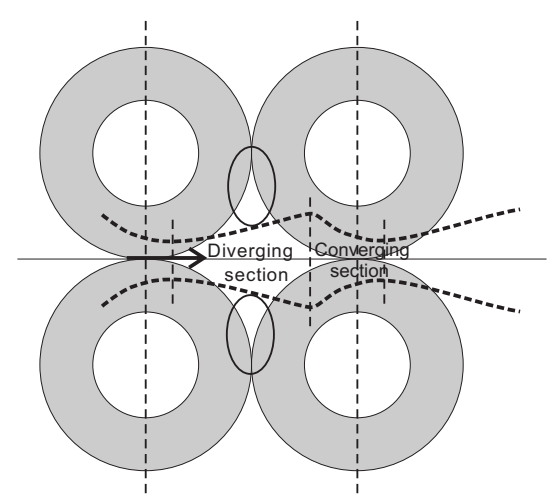

FIG. 7. Illustration of the active core (dashed line) and stagnant fluid (ellipse). (a)

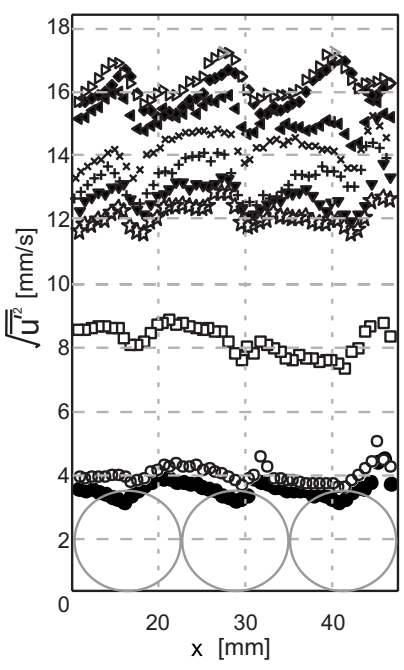

FIG. 8. (a) Turbulence intensities intensities.

is likely to become unstable and break up. In the following text we further explore this model by investigating various velocity statistics.

\section{B. Higher order velocity statistics}

The second, third, and fourth order moments of velocity were calculated according to the following expressions:

$$
\begin{aligned}
& \sigma_{u}=\left\langle\sqrt{\overline{u^{\prime 2}}}\right\rangle, \\
& M_{\text {иии }}=\left\langle\frac{\overline{u^{\prime 3}}}{\sigma_{u}^{3}}\right\rangle, \\
& K_{u}=\left\langle\frac{\overline{u^{\prime 4}}}{\sigma_{u}^{4}}\right\rangle,
\end{aligned}
$$

where $\sigma_{u}$ is the streamwise turbulence intensity, $M_{\text {иии }}$ is the streamwise skewness, $K_{u}$ is the streamwise kurtosis, and $u^{\prime}$ is the instantaneous velocity fluctuation from the temporal mean. Overbars denote time-averaged variables and \langle\rangle denote spatially averaged variables.

Figures $8(a)$ and $8(\mathrm{~b})$ are plots of the turbulence intensity based on time-averaged and double-averaged variables, respectively. The local minima and maxima in the profiles shown in Fig. 8(a) are at locations which are opposite to those of the velocity profiles: minimum velocity corresponds to maximum turbulence intensity and vice versa. This is not surprising as the minimum velocity marks the end of the flow-diverging section where turbulence is likely to be enhanced, while the maximum velocity occurs at the end of flow convergence, which is known to suppress turbulence. In absolute terms this difference is very pronounced at higher velocities, whereas at low velocities turbulence intensity is nearly constant. In relative terms (scaled with the mean velocity) turbulence intensity remains practically constant for all experiments. 
(a)

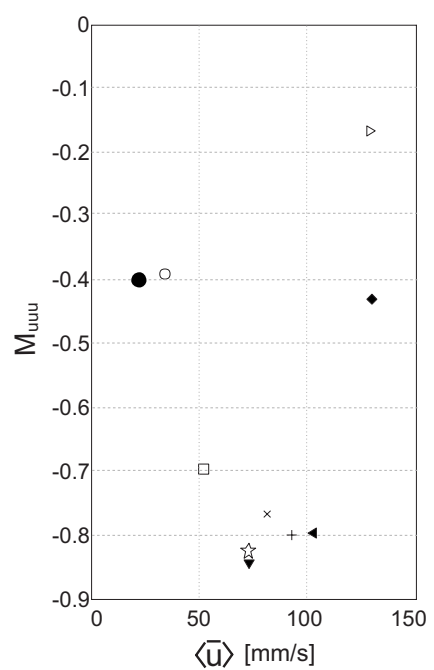

(b)

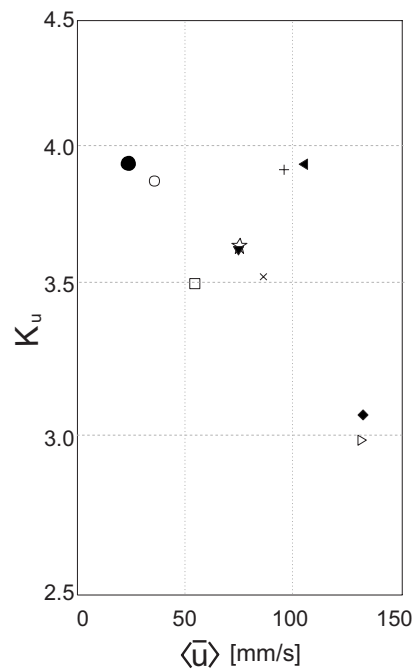

FIG. 9. (a) Spatially averaged skewness. (b) Spatially averaged kurtosis.

The third order velocity moment, or skewness, is plotted in Fig. 9(a). There are three clearly identifiable regions in this graph. The first two tests with lowest Re and the last two with highest Re have much smaller (in absolute terms) skewness of around -0.4 , than the medium Re experiments where the values are between -0.8 and -0.9 . Figure $9(\mathrm{~b})$ presents the spatially averaged kurtosis. Kurtosis $\left(K_{u}\right)$ is a measure of the level of intermittency of the turbulence. It is only the two high $\mathrm{Re}$ experiments that have a value corresponding to a Gaussian distribution, whereas for all other tests the kurtosis ranges from approximately 3.5 to 4.0 .

From the skewness data in Fig. 9(a), it is clear that a transition of flow properties occurs twice, first between Reynolds numbers 110 and 170 and second between 345 and 440. These transitions are not visible in the kurtosis results except for the fact that the data are more scattered for medium Reynolds numbers.

Spatial variability of time-averaged velocities can be quantified using the differences between the local timeaveraged velocities and their corresponding double-average, $\widetilde{\bar{u}}$, i.e., $\bar{u}=\langle\bar{u}\rangle+\widetilde{\bar{u}}$. These departures $(\widetilde{\bar{u}})$ are strongly influenced by the pore shape (form) and are therefore called form-induced velocity disturbances, or simply form-induced velocities. They appear as a result of spatial-averaging in the same way as velocity fluctuations at a point are the product of time averaging. Further details about double-averaging methodology can be found in Ref. 8 .

Spatial averaging of the time-averaged momentum equation produces an additional term called form-induced stress (also dispersive stress in some papers). It results from spatial averaging in a manner which is analogous to the Reynolds stress resulting from time averaging. The normal component of the form-induced stress for the streamwise direction is $-\rho \tilde{\bar{u}} \tilde{\bar{u}}$. Profiles of (kinematic) form-induced momentum flux, $\tilde{u} \tilde{u}$ and form-induced stress, $\langle\tilde{\bar{u}} \widetilde{\bar{u}}\rangle$ are plotted in Figs. 10(a) and $10(\mathrm{~b})$. For clarity only the profiles for tests with velocities 20.48, 74.36, and $132.40 \mathrm{~mm} / \mathrm{s}$ are plotted in Fig. 10(a). From this figure it is clear that form-induced momentum flux

(a)

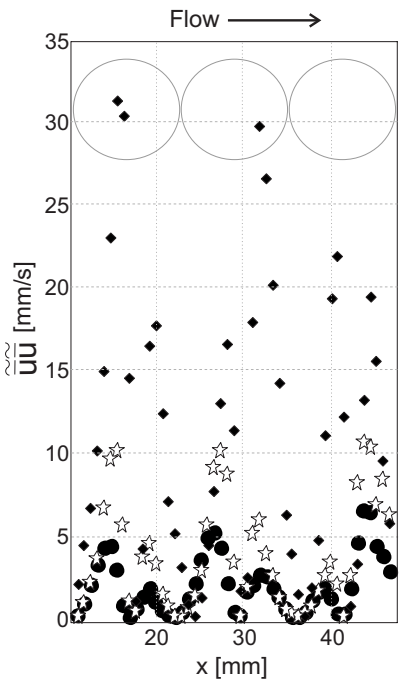

(b)

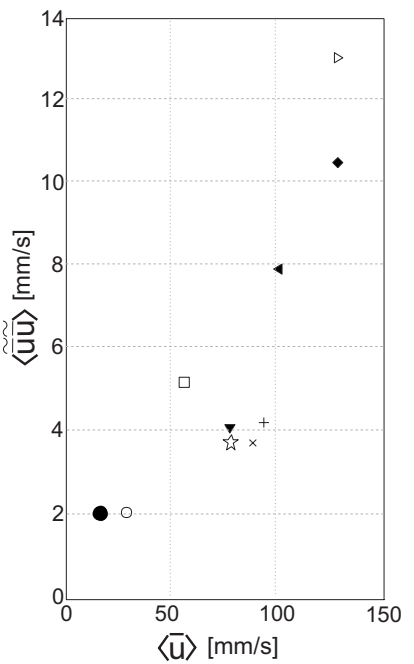

FIG. 10. (a) Form-induced momentum flux. (b) Form-induced stress.

is far more regular for the lower Reynolds number tests. The magnitude of the form-induced stress shown in Fig. 10(b) increases rapidly for higher Re tests.

\section{Two-point statistics}

To further explore the different flow regimes detected in the one-point velocity statistics, two-point correlation coefficients were calculated according to the following expression (Roy et $a l .{ }^{9}$ ):

$$
R_{u u}(\delta x, \tau)=\frac{\overline{u^{\prime}\left(x_{1}, t-\tau\right) u^{\prime}\left(x_{2}, \tau\right)}}{\sigma_{u 1} \sigma_{u 2}},
$$

where $x_{1}$ and $x_{2}$ are the locations of the velocity measurements along the pore, $\delta x=x_{2}-x_{1}$, and $\tau$ is the time lag.

The distance $\delta x$ was always a multiple of the bead diameter so that points $x_{1}$ and $x_{2}$ had an identical position relative to the bead geometry. Comparison between the results for two extreme spatial positions, one at the velocity maxima ( $2 \mathrm{~mm}$ downstream of the narrowest section of the pore throat) and another at the velocity minima (3 $\mathrm{mm}$ downstream of the widest section) showed negligible difference. For this reason only the former is presented here: Point one is just behind the pore throat and the distances are $\delta x=12$, 24, 36, and $48 \mathrm{~mm}$.

Figure 11 shows the two-point correlation coefficients for all experiments, calculated with zero time lag, $\tau=0$, and variable $\delta x$. Again they form three distinct groups. The low Re tests (left graph) are different from the rest because they do not fully decorrelate over the entire measurement window. This implies regular periodic motion, which has the same phase along the whole measurement window, such as the pulsating unsteady-laminar flow described in Ref. 3. In contrast with this, the medium Re experiments have decorrelation lengths shorter than or equal to one bead diameter. This indicates that the laminar flow was completely disrupted by disturbances generated at the separation point of the core flow. The spatial scale of these disturbances is 
(a)

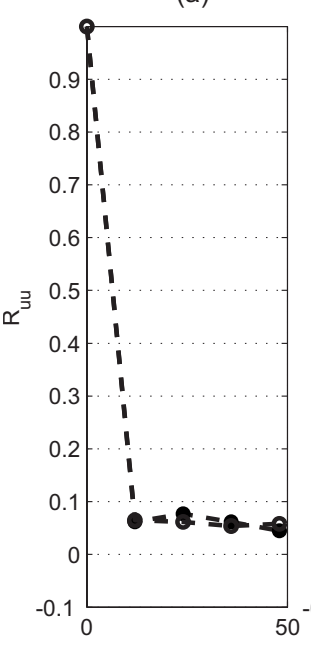

(b)

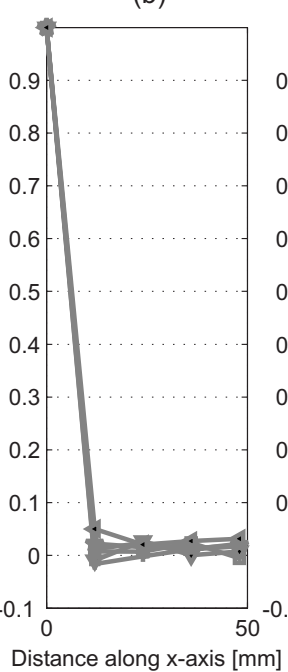

(c)

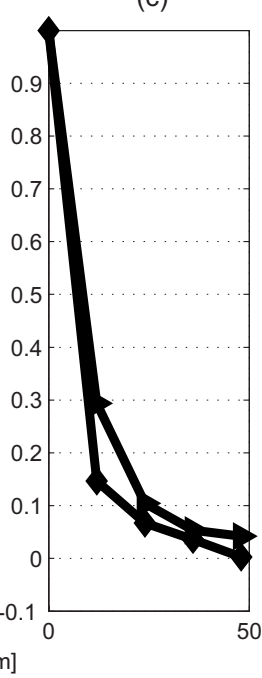

FIG. 11. Two-point correlations ( $\tau=0$ and variable $\delta x$ ); (a) low momentum (thin black); (b) "medium" momentum (gray); (c) high momentum (thick black).

smaller than a bead diameter. For the two high Re experiments the correlation coefficients gradually approach zero, indicating the presence of turbulent events of various spatial scales, i.e., fully developed turbulent flow.

Figures 12 and 13 present the correlation coefficients
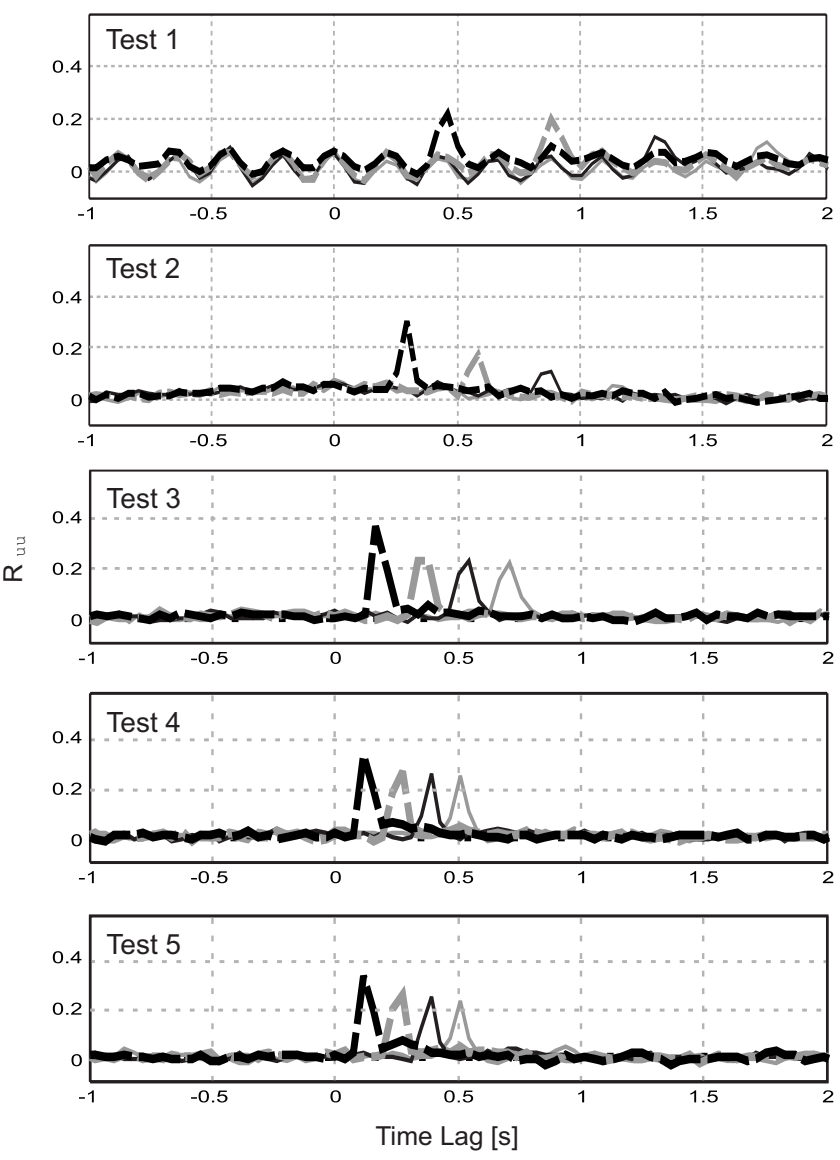

FIG. 12. Tests 1-5: two-point correlation data. $\delta x=12 \mathrm{~mm}$ (dashed black line), $24 \mathrm{~mm}$ (dashed gray line), $36 \mathrm{~mm}$ (solid black line), and $48 \mathrm{~mm}$ (solid gray).
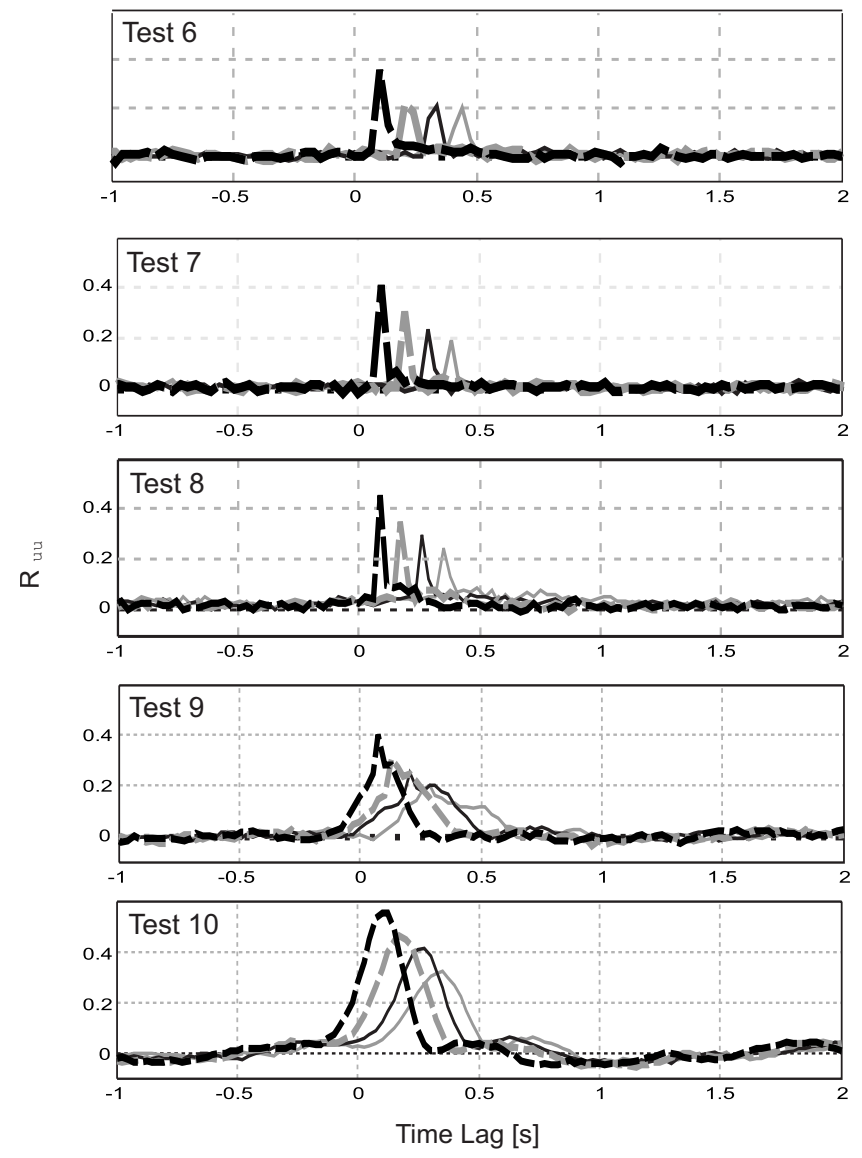

FIG. 13. Tests 6-10: two-point correlation data. $\delta x=12 \mathrm{~mm}$ (dashed black line), $24 \mathrm{~mm}$ (dashed gray line), $36 \mathrm{~mm}$ (solid black line), and $48 \mathrm{~mm}$ (solid gray).

calculated with fixed spatial lags and a variable time lag of between -1 and +2 s. Test 1 with the smallest Reynolds number shows a distinct periodic shape along the whole analyzed range of time lags. This confirms the previous suggestion that periodic unsteady-laminar motions exist to a certain extent, and are associated with the pulsations of the inertial core described in Ref. 3. The period of this motion-of around $0.2 \mathrm{~s}-$ is clearly visible in the graph. In addition to this pulsating motion which is in phase across the whole measurement window, equally regular disturbances exist, which are translated downstream.

Test 2 shows somewhat different features: persistent periodic motion has been broken up, there is only a very slight trace of it in the graphs. The only prominent features are the regular disturbances translated downstream. They are somewhat different from those detected for test 1 in that their time base is narrower and their dissipation is more thorough. This might indicate that these disturbances have managed to form vortices.

All medium Reynolds number tests (3-8) show almost identical properties. Local peaks have a narrow time base and are translated downstream at constant speed. The difference between this and test 2 is that now we have a slightly higher correlation coefficient; $R_{u u} \approx 0.22$ in test 3 compared 


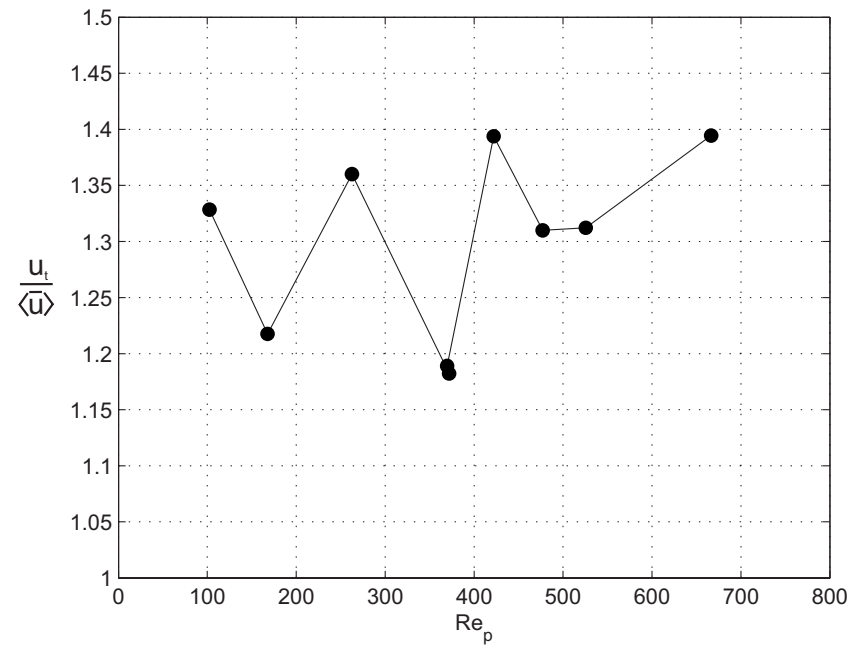

FIG. 14. Ratio of the convection velocity, $u_{t}$ to the double-averaged velocity, $\langle\bar{u}\rangle$.

with $R_{u u} \approx 0.18$ in test 2 . This is the result of more coherent motions with narrow, well-defined spatial and temporal scales.

Test 9 shows a transition between tests $3-8$ and test 10 : A distinct sharp peak is still present, but it emerges from a Gaussian-shaped graph, which becomes the dominant feature of test 10 . The change in the shape is due to the onset of fully developed turbulent flow involving coherent structures with a large range of scales. Another interesting feature of test 10 is that the overall periodicity reappears, however it is less regular, more pronounced for positive time lags, and has a much larger period. This periodicity is due to the turbulent fluctuations of the "pipelike" core flow.

Comparing the magnitudes of the distinct peaks in the correlation graphs provides the information on the longevity of flow structures. Two different patterns can be identified in Figs. 12 and 13: For low Re tests (1 and 2) and high Re tests (7-10) the peaks gradually decrease throughout (from $\delta x$ $=12 \mathrm{~mm}$ until $\delta x=48 \mathrm{~mm}$ ), while for medium Re tests (3-6) the very first $\delta x=12 \mathrm{~mm}$ peak is somewhat higher, while the others are approximately constant. The gradually decreasing peaks in the low Re tests indicate the "breaking up" and viscous dissipation of flow disturbances. For the medium Re tests there are some short-lived structures which are dissipated by the action of viscous stress, between $\delta x$ $=12 \mathrm{~mm}$ and $\delta x=24 \mathrm{~mm}$. However the majority of the structures persist until $\delta x=48 \mathrm{~mm}$. These tests relate to a range of flow regimes, where the majority of the generated coherent motions are of sufficient strength to persist over a relatively long temporal and spatial window. This is because turbulence is not well enough developed to cause their breakdown. Finally, the gradually decreasing peaks in the high Re tests are due to the conventional eddy cascade of fully developed turbulence.

The time lags associated with peak correlation were used to calculate the translational (or convection) velocity $\left(u_{t}\right.$ $=\delta x / \delta t)$. The ratio of the convection velocity to the pore velocity is shown in Fig. 14. From the figure it is clear that in all tests flow disturbances are translated more rapidly than (a)

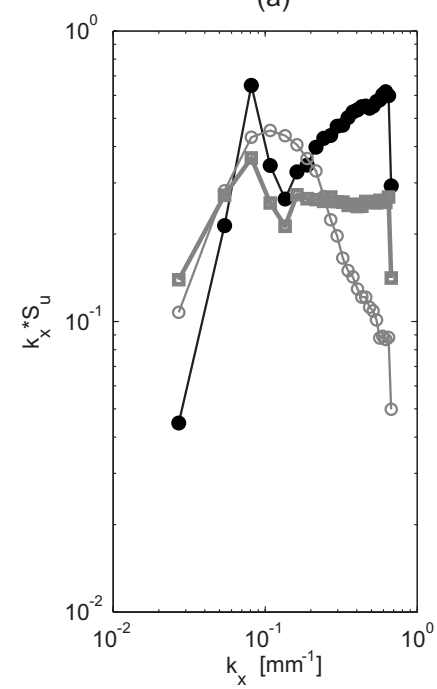

(b)

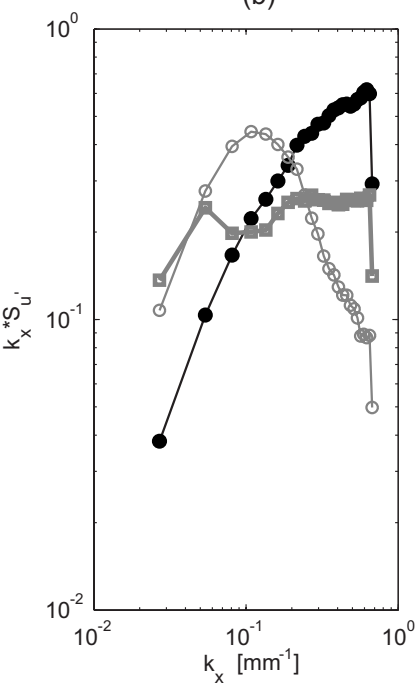

FIG. 15. Velocity spectra in the wave number domain for low $\operatorname{Re}$ (test 1 , black), medium Re (test 5, gray), and high Re (test 10, white).

the double-averaged pore velocity. The convection velocity in several of the lower momentum tests is approximately $15 \%$ greater than that of the bulk flow. The convection velocity increases to nearly $140 \%$ of the bulk flow velocity in some of the higher momentum tests. This is probably due to the velocity distribution across the pore and means that the flow disturbances are mainly transported by the highest fluid velocity around the pore center, rather than at the average cross-sectional velocity measured by the UVP.

\section{Velocity spectra}

In this study, the deployment of the UVP probe allowed instantaneous measurements of $u$ at 128 points along the $x$ direction and this made it possible to compute spectra directly in the wave number domain. This represents a significant advantage since, as shown above, Taylor's hypothesis cannot be used to obtain information on the spatial structure of turbulence from the UVP measurements. Spectra in the wave number domain were computed as follows: First instantaneous spectra $S_{u}\left(k_{x}\right)$ were calculated for each instantaneous profile of velocity $u(x)$. They were then time averaged for each wave number to make the confidence interval for $S_{u}\left(k_{x}\right)$ smaller at each $k_{x}$. This procedure was used twiceonce with the whole instantaneous velocities and once with velocity fluctuations. This resulted in two kinds of spectra: $S(u)$ and $S\left(u^{\prime}\right)$, respectively.

Figure 15 shows a comparison of velocity spectra for low, medium and high Reynolds number tests. They are presented in premultiplied form, i.e., the vertical coordinates show $k_{x} S_{u}\left(k_{x}\right)$ and $k_{x} S_{u^{\prime}}\left(k_{x}\right)$. The former spectra, calculated for the whole velocity $(u)$, show a distinct peak corresponding to $12 \mathrm{~mm}$, i.e., the wavelength of one bead diameter. This peak, which is obviously caused by the periodicity induced by the pore geometry, exists for low and medium Reynolds numbers, but disappears in the high Re test. This indicates the presence of a wide range of other, more energetic, spatial scales which are sufficient to mask the peak. 

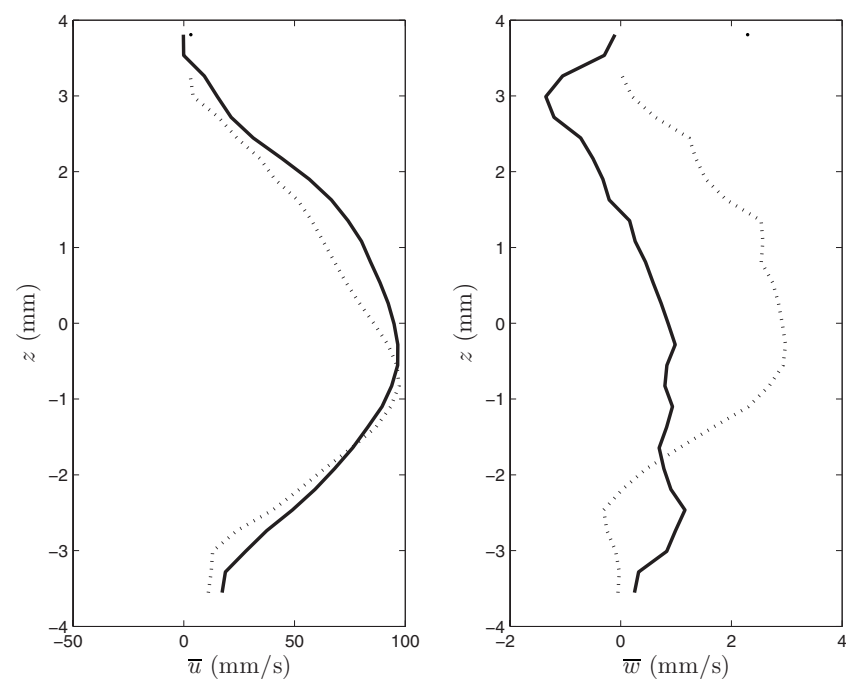

FIG. 16. Profiles of time-averaged streamwise, $\bar{u}$ and bed normal, $\bar{w}$ velocity for the PIV test. The figure shows the profile in the upstream pore (dashed line) and downstream pore (solid line).

The geometry-induced peak is absent from all spectra based on velocity fluctuations, $k_{x} S_{u^{\prime}}\left(k_{x}\right)$. The low Re test shows that the maximum energy is concentrated at the smallscale end of the spectrum. In the medium Re test some of the energy has moved from the small-scale end toward the midand large scales, resulting in a relatively even (compared to the other two cases) distribution across the whole range of scales. The high Reynolds number test has a much narrower range of dominant scales covering the interval between approximately 5 and $12 \mathrm{~mm}$.

\section{E. Spatial flow structure across the pores}

In this section we present the PIV measurements of velocity across the central section of the pore, in the middle of the gap where the view is not obstructed by the spheres. This visible section is the diamond-shaped gap between the four adjacent spheres of Fig. 7. The measurement window covered two adjacent gaps.

Figure 16 shows the profiles of time-averaged streamwise velocity, $\bar{u}$ and bed-normal velocity, $\bar{w}$ along the center of the upstream (dashed line) and downstream (solid line) gaps. The profiles were calculated along the widest pore section. The figure shows that the $\bar{u}$ profiles measured in the two adjacent gaps are very similar. The height of the profile where the streamline velocity is greater than zero is about 6 $\mathrm{mm}$, confirming that the active pore section is contained to the central core. The $\bar{w}$ profiles for the two gaps show poorer agreement, which is probably due to slight variations of the pore geometry between the adjacent gaps.

Streamwise and bed-normal turbulence intensity profiles are shown in Fig. 17. Both streamwise and bed-normal turbulence intensity are approximately constant across the gap. Although lateral velocity is smaller than the streamwise velocity by an order of magnitude, the associated turbulence intensities are comparable.

The consistency between the UVP and PIV measurements was investigated by calculating two-point correlation
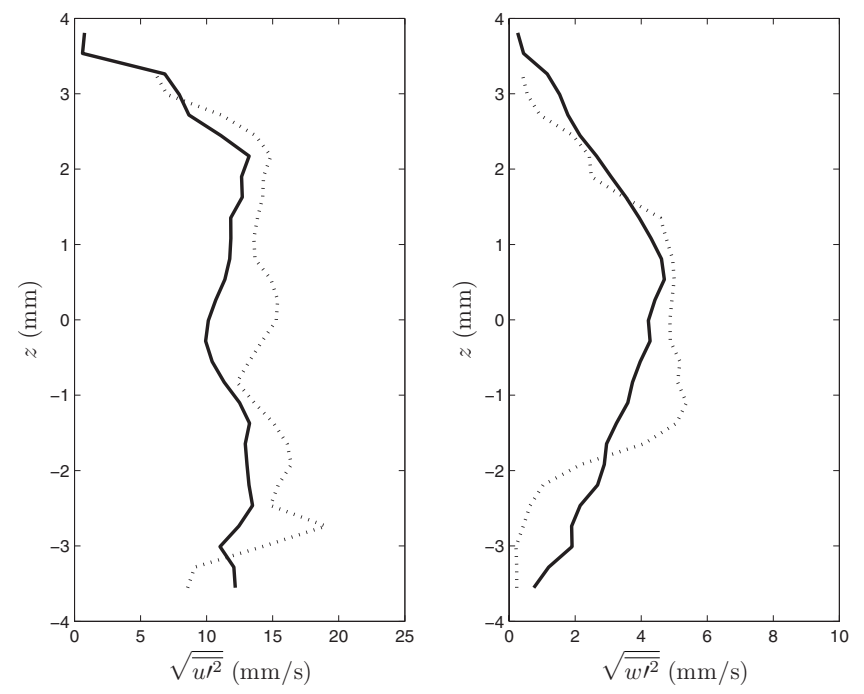

FIG. 17. Profiles of time-averaged streamwise, $\sqrt{\overline{u^{\prime 2}}}$ and bed normal, $\sqrt{\overline{u^{\prime 2}}}$ turbulence intensity for the PIV test. The figure shows the profile in the upstream pore (dashed line) and downstream pore (solid line).

coefficients, in a similar manner to that presented for the UVP results. The instantaneous velocity profiles measured along the same central axes as for Figs. 16 and 17 were averaged over the profile height. This resulted in a time series of cross-sectional velocities, $u_{1}$ and $u_{2}$ for the upstream and downstream profiles, respectively. Thus the distance between the two profiles, $\delta x$ was $12 \mathrm{~mm}$ while the time lag was varied as before.

Figure 18 shows results for both the streamwise and lateral velocity fluctuations. The profiles of $R_{u u}$ and $R_{w w}$ reach a maximum at $\tau=0.15$ and $0.22 \mathrm{~s}$, respectively. The shape of the correlation profiles agree very well with the corresponding UVP results for a Reynolds number of 340. The results suggest that this PIV test is at the higher end of the transition region between laminar and fully developed turbulent flow.

From the UVP measurements it was inferred that above approximately $\operatorname{Re}_{p}=170$ turbulence begins to develop. The

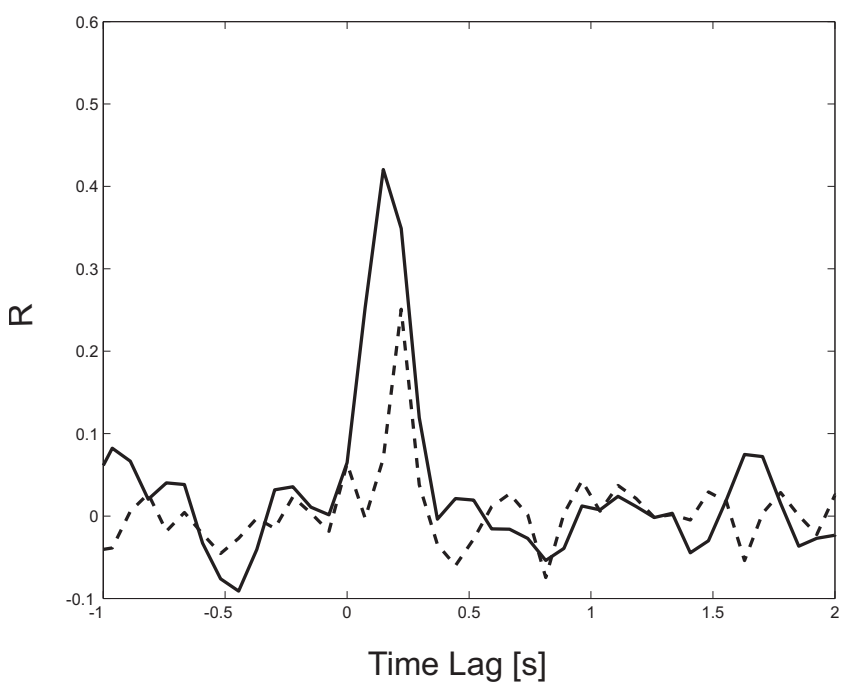

FIG. 18. Two-point correlation coefficients, $R_{u u}$ (solid line) and $R_{w w}$ (dotted line) calculated with $\delta x=12 \mathrm{~mm}$ and a varying time lag, $\tau$. 
(a)

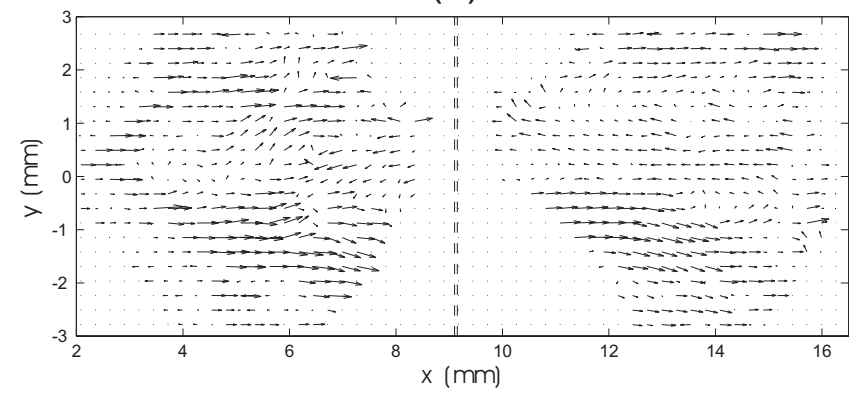

(b)

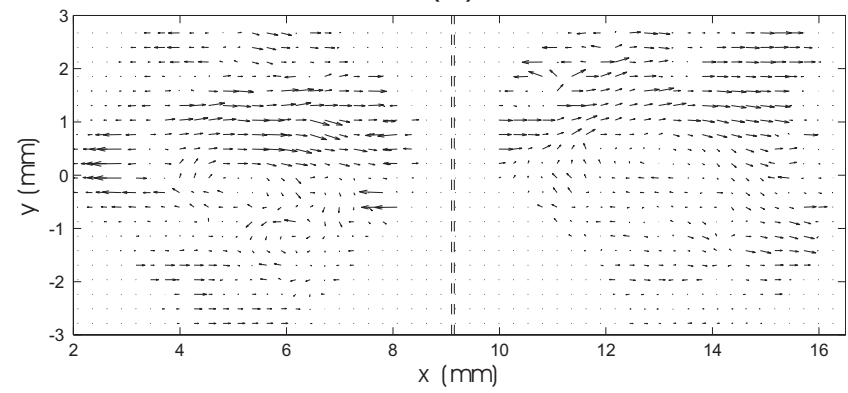

(c)

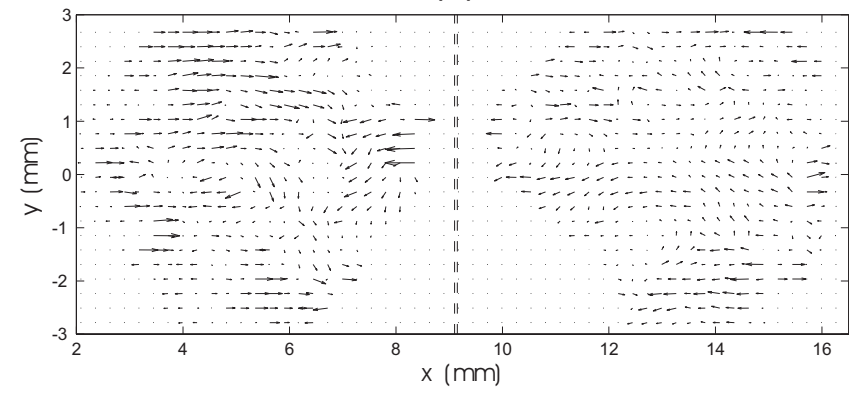

FIG. 19. [(a) and (b)] Instantaneous velocity fluctuations during positive $\left(u^{\prime}>0\right)$ turbulent events. Flow is from left to right. The right window has a $0.15 \mathrm{~s}$ time lag compared to the left window.

correlation data for both the UVP and PIV experiments reinforce this conclusion. Further analysis was performed with the original velocity data for the upstream pore, $u_{1}^{\prime}(t)$ and the velocity shifted by a time lag of $0.15 \mathrm{~s}$ in the downstream pore, $u_{2}^{\prime}(t+0.15 \mathrm{~s})$. As shown above, this time lag gave a maximum correlation for the profiles of velocity fluctuations, $u_{1}^{\prime}$ and $u_{2}^{\prime}$. These two velocity fields were conditionally sampled, in order to identify significant turbulent events. The criteria for conditional sampling was $u_{1}^{\prime}+u_{2}^{\prime}>$ threshold, for "positive" events, and $u_{1}^{\prime}+u_{2}^{\prime}<-$ threshold, for "negative" events. Some vector maps of instantaneous velocity fluctuations during the positive and negative events are presented in Figs. 19 and 20, respectively. All vector maps show distinct regions of coherent motion at various locations within the upstream and downstream pores.

Finally, using the same sampling criteria an ensemble average of the flow field of the 15 largest positive and 15 largest negative events is shown in Fig. 21. The figure shows that the most energetic events involve the simultaneous streamwise movement of fluid over the majority of the pore (a)

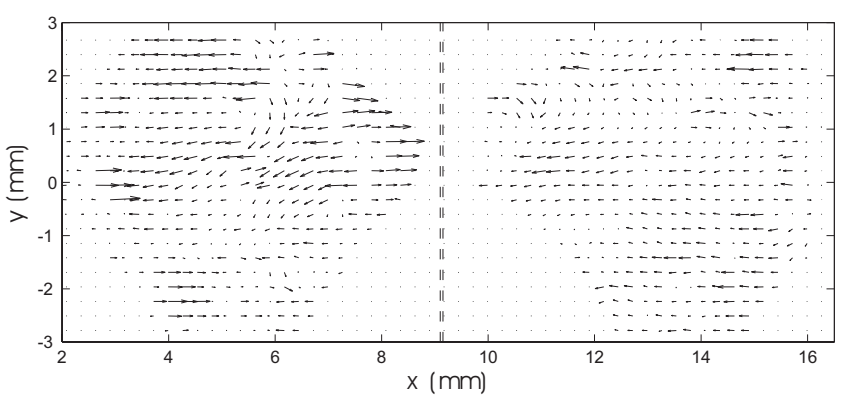

(b)

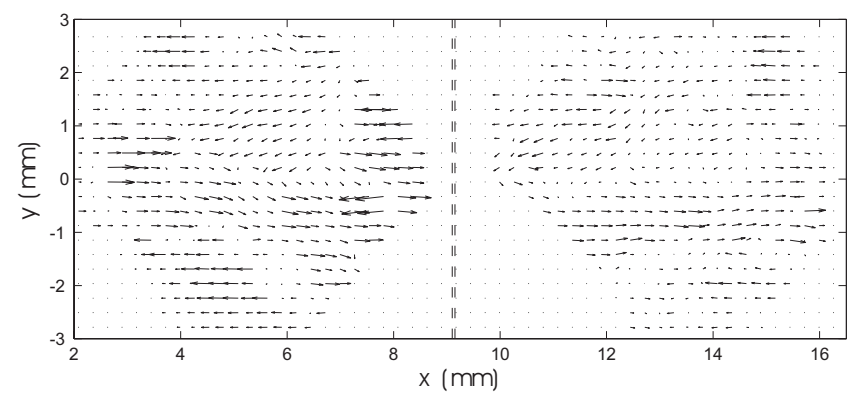

(c)

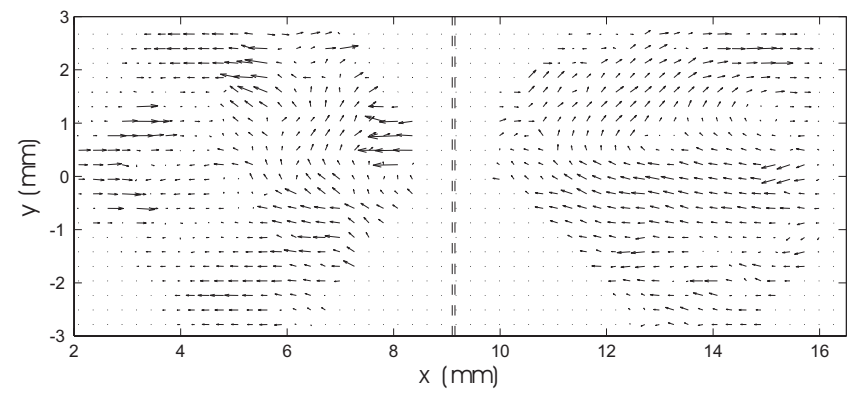

FIG. 20. [(a) and (b)] Instantaneous velocity fluctuations during negative $\left(u^{\prime}<0\right)$ turbulent events. Flow is from left to right. The right window has a $0.15 \mathrm{~s}$ time lag compared to the left window.

space, accompanied by some lateral motion near the perimeter of the inertial core and close to the pore throats.

\section{CONCLUSIONS}

We presented experimental velocity data measured in the pore of a regular porous medium, which was formed using uniform diameter spheres packed in a cubic pattern. The experimental methodology allowed the measurement of the average pore velocity at a series of points along the pore. The results suggest the existence of the following three flow regimes: unsteady-laminar, transitional, and turbulent flows. This is consistent with the findings of Dybbs and Edwards, ${ }^{3}$ which were based mainly on flow visualization. The data presented in this paper provide further information on the velocity statistics and spatial scales, which can be used in their own right and also for the validation of numerical models.

The time-averaged pore velocity has a wavy streamwise variation, revealing regions of convergence and divergence of the active flow core within the pore. The turbulence inten- 
(a)

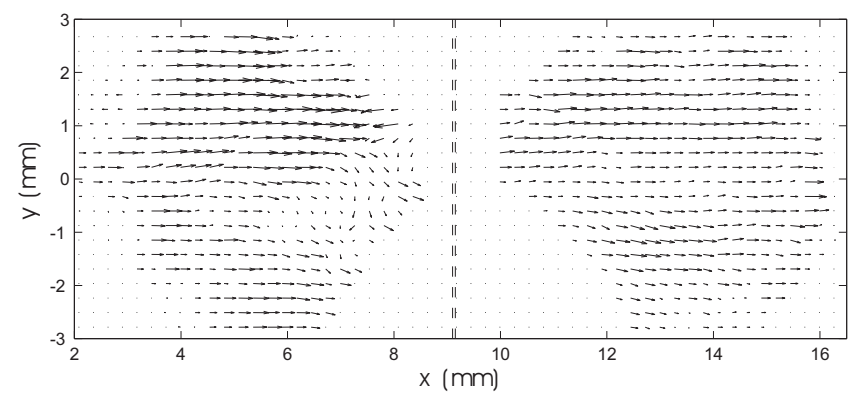

(b)

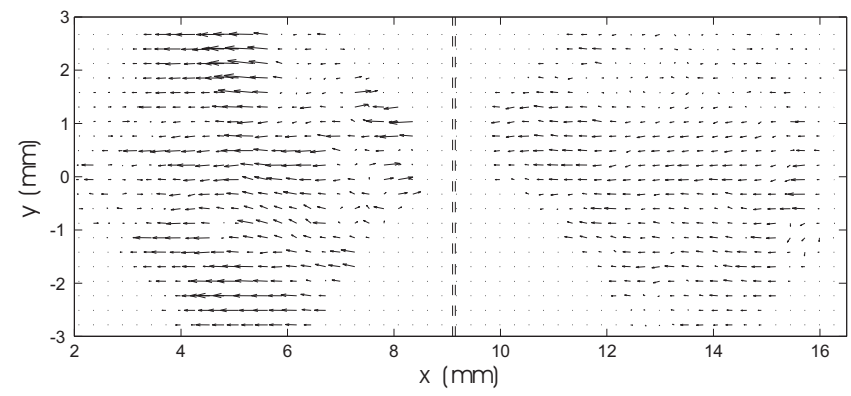

FIG. 21. [(a) and (b)] Composite average of the 15 most energetic positive $\left(u^{\prime}>0\right)$ turbulent events (top) and negative $\left(u^{\prime}<0\right)$ events (bottom). Flow is from left to right. The right window has a $0.15 \mathrm{~s}$ time lag compared to the left window.

sity profiles have a local maxima where the velocity is low, suggesting that the highest turbulence occurs when the diverging flow hits a downstream obstacle.

The different characteristics of the three flow regimes become apparent through the third order velocity moments. All three regimes have negative skewness but the absolute values are much larger in the transitional regime than in the other two. The prevalence of slower-than-average velocities is the result of the relatively infrequent occurrence of very energetic events, which raise the overall average velocity but do not affect the, still relatively stable, bulk flow. Once turbulence is fully developed high-momentum coherent motions occur more frequently. The kurtosis data reinforce this conclusion. The kurtosis data indicate that flow intermittency decreases from unsteady laminar, through transitional to the fully developed turbulent flow regime.

The two-point velocity statistics calculated for the UVP measurements reveal a standing-wave-like behavior of the unsteady-laminar flow regime. This is followed by the occurrence of infrequent events with very well-defined time scales, which are the signature of the transitional regime. Finally the presence of the whole range of scales characterizes fully developed turbulence. This is further confirmed by the spatial velocity spectra which show the shifting of turbulent energy toward lower wave numbers as the flow regime changes from unsteady laminar, through transitional into fully developed turbulence.

The eddy convection velocities are higher, by approximately $30 \%$, than the mean flow velocities. This suggests that coherent structures are transported at the maximum ve- locity in the pore cross section, i.e., by the velocity at the pore's center.

The velocity and turbulence intensity profiles across the widest pore cross section were found from the PIV experiment. The velocity profiles suggest that the active fluid motion covers only the central core of the pore. The lateral turbulence intensity has a similar magnitude to the streamwise intensity.

The PIV recorded a series of instantaneous velocity fields in the two adjacent gaps between the spheres. Twopoint statistics calculated for the central profiles in these gaps show a very similar pattern to those found from the UVP data for a similar Reynolds number. The time lag which produced maximum correlation was used to offset the instantaneous velocity field in the downstream gap and search for significant events in those two fields. These events were found through conditional sampling, based on the fluctuations of the cross-sectional velocity in both gaps. The instantaneous velocity field recorded during these significant events shows the presence of coherent flow structures.

In summary, the three flow regimes within the pores of the regular porous medium have the following characteristics:

(1) Unsteady laminar flow. The inertial core undergoes periodic pulsations. The velocity distribution is slightly skewed toward the low-momentum end.

(2) Transitional flow. Coherent motions with well-defined very narrow spatial scales and frequency occur occasionally, while the bulk flow remains at low velocities so that the negative skewness is enhanced.

(3) Turbulent flow. Coherent motions with a range of spatial and temporal scales are shed at the boundary of the pipelike core flow. The negative skewness and flow intermittence are both reduced. The turbulent kinetic energy is contained in a range of spatial scales.

Engineering applications involving porous media flows rely on our ability to predict flow characteristics under different working conditions. Numerical models are often used to make such predictions. The results presented in this paper can be used in their own right to better understand the range of microscopic flow conditions, which belong to the broad category of the macroscopic Forchheimer flow regime. The results can also be used for the validation of numerical models related to transitional and turbulent porous media flows.

\section{ACKNOWLEDGMENTS}

The authors are grateful to the anonymous reviewer for all insightful comments and valuable suggestions.

${ }^{1}$ S. Ergun, "Fluid flow through packed columns," Chem. Eng. Prog. 48, 89 (1952).

${ }^{2}$ J. Ward, "Turbulent flow in porous media," J. Hydr. Div. 90, 1 (1964).

${ }^{3}$ A. Dybbs and R. Edwards, "A new look at porous media fluid mechanics (Darcy to turbulent)," Fundamentals of Transport Phenomena in Porous Media (Martinus Nijhoff, Dordrecht, 1984).

${ }^{4}$ R. Fand, B. Kim, A. Lam, and R. Phan, "Resistance to flow of fluids 
through simple and complex porous media whose matrices are composed of randomly packed spheres," ASME J. Fluids Eng. 109, 268 (1987).

${ }^{5}$ D. Seguin, A. Montillet, and J. Comiti, "Experimental characterization of flow regimes in various porous media-1: Limit of laminar flow regime," Chem. Eng. Sci. 53, 3751 (1998).

${ }^{6}$ I. Kececioglu and Y. Jiang, "Flow through porous media of packed spheres saturated with water," ASME J. Fluids Eng. 116, 164 (1994).
${ }^{7}$ C. Manes, D. Pokrajac, I. McEwan, V. Nikora, and L. Campbell, "Application of UVP within porous beds," J. Hydraul. Eng. 132, 983 (2006).

${ }^{8}$ M. de Lemos, Turbulence in Porous Media: Modeling and Applications (Elsevier, New York, 2006), Vol. 1.

${ }^{9}$ A. Roy, T. Buffin-Belanger, H. Lamarre, and A. Kirkbride, "Size, shape and dynamics of large-scale turbulent flow structures in a gravel-bed river," J. Fluid Mech. 500, 1 (2004). 